\title{
Um Modelo para Integração e Disponibilização de Dados na Área de Saúde Governamental
}

\author{
Alternative Title: A Model for Data Integration and Availability in Health \\ Government Area
}

\author{
Ana Carolina C Ferronato \\ Universidade Federal \\ Fluminense \\ Rio das Ostras, RJ \\ accferronato@id.uff.br
}

\author{
Fernanda Ramos Pires \\ Universidade Federal \\ Fluminense \\ Rio das Ostras, RJ \\ fpires@id.uff.br
}

\author{
Flavia Cristina Bernardini \\ Universidade Federal \\ Fluminense \\ Rio das Ostras, RJ \\ fcbernardini@id.uff.br
}

\begin{abstract}
RESUMO
Um dos caminhos de evolução de cidades inteligentes vão na direção de uma forte integração de todas as dimensões da inteligência - humana, coletiva e artificial - disponíveis em uma cidade. Em busca de auxiliar nesta evolução, o presente trabalho apresenta o mapeamento de um modelo de dados a fim de criar uma ontologia para cidades inteligentes brasileiras na área de saúde governamental, aplicando conceitos de Web semântica e integração de dados, para buscar soluções para problemas de alinhamento entre base de dados.
\end{abstract}

\section{Palavras-Chave}

OWL; RDF; Ontologia; Web Semântica; E-cidade; Protégé

\begin{abstract}
One of the evolution paths of smart cities go towards a strong integration of all intelligence dimensions - human intelligence, collective and artificial - available in a city. In pursuit of helping this evolution,this paper presents the mapping of a data model in order to create an ontology for Brazilian smart cities in government health care, by applying Semantic Web concepts and data integration, to seek solutions to alignment problems between databases.
\end{abstract}

\section{Categories and Subject Descriptors}

H.3 [Information Storage and Retrieval]: Online Information Services-Data sharing; K.6 [Management of Computing and Information Systems]: Project and People Management-Strategic information systems planning; J.1 [Administrative Data Processing]: Government

\section{General Terms}

Data Integration; Electronic Government; Smart City

Permission to make digital or hard copies of all or part of this work for personal or classroom use is granted without fee provided that copies are not made or distributed for profit or commercial advantage and that copies bear this notice and the full citation on the first page. To copy otherwise, to republish, to post on servers or to redistribute to lists, requires prior specific permission and/or a fee.

SBSI May 17th-20th, 2016, Florianópolis, Santa Catarina, Brazil

Copyright SBC 2016

\section{Keywords}

OWL; RDF; Ontology; Semantic Web; E-cidade; Protégé

\section{INTRODUÇÃO}

O conceito de Cidade Inteligente está embasado em seis principais dimensões: economia, mobilidade, ambiente, pessoas, vida e governança inteligentes [9]. Define-se uma cidade como inteligente quando seus investimentos em capital humano e social, em transporte urbano e infraestrutura de TIC alimentam o desenvolvimento econômico sustentável e uma melhor qualidade de vida, com sábio gerenciamento de recursos naturais, através do governo participativo [7]. O conceito de Cidade Inteligente é distinto de ideias similares como Cidade Digital pois o conceito de Cidade Inteligente foca em fatores tais como capital humano e educação como guia do crescimento urbano, enquanto que as outras ideias focam na infraestrutura das TICs [4]. Devido à definição de computação ubíqua, i.e., a onipresença da informática e computação no cotidiano das pessoas, definições de Cidade Ubíqua têm sido propostas. Daí vem o estágio inicial de uma Cidade Inteligente, que provê serviços combinados entre a infraestrutura de TI e as unidades, ou empresas, prestadoras de serviço na cidade [9]. Lee et al. [10] define uma Cidade Inteligente em termos da convergência dos serviços de TI com o espaço urbano de tal maneira que os cidadãos possam acessar serviços inteligentes independentemente de tempo ou lugar. Em resumo, uma Cidade Inteligente deve prover serviços aos seus cidadãos via sua infraestrutura baseada em tecnologias de TICs. Na prefeitura da cidade de Rio das Ostras, por exemplo, as bases de dados são distribuídas entre as diversas secretarias. Tais bases de dados não são integradas, e ainda há problemas de alinhamento entre as bases de dados, ou seja, não há um identificador único (como o CPF - Cadastro de Pessoa Física) para as mesmas entidades representadas em todas as bases, segundo o subsecretário de TI do município [11]. Uma solução para este problema é adotar um sistema integrado em todas as secretarias, a exemplo do software público E-Cidade [13]. Assim, utilizar uma ontologia pode auxiliar a guiar o processo de alinhamento nas bases de dados. Esse software auxilia na automatização de diversas atividades nas prefeituras. No entanto, mesmo o E-Cidade tendo muitos módulos, nem todo município adota esse software, e nem toda secretaria de um município o adota (mesmo que outras secretarias o adotem), 
devido a algumas limitações no sistema [11]. Daí, outra possível solução é construir uma ontologia para integração e alinhamento dessas bases de dados. Encontrar especialistas disponíveis para auxiliar no processo de construção dessa ontologia, cuja entidade principal é o cidadão, e que deve abordar o cidadão em muitos serviços por ele utilizado no município, é muito difícil, já que diversos domínios são abordados - saúde, educação, transporte, planejamento, dentre outros. Em [6] é definida uma ontologia genérica e reusável para representar definições de Indicadores Globais de Cidades e sua instanciação na Web Semântica pelas cidades. A ontologia combina, adapta e estende ontologias de fundamentação existentes.

O objetivo deste trabalho é apresentar um modelo RDFschema da área de saúde, que futuramente servirá de base para a ontologia cujo conhecimento diz respeito a todos os serviços relacionados a saúde que um cidadão pode utilizar numa cidade. Dados do cidadão devidamente integrados podem auxiliar na previsão de serviços, regiões que requerem mais investimentos em áreas específicas, dentre outros. Esse Esquema RDF foi construído utilizando como base a ontologia proposta em [6] para dar início à construção dessa nova ontologia, e foi reutilizado o conhecimento obtido na construção do software E-cidade, por meio da análise da base de dados do sistema.

Este trabalho está dividido como segue: na seção 2 são descritos os trabalhos relacionados a cidades inteligentes.Na seção 3 são descritas ferramentas utilizadas para o desenvolvimento deste trabalho, são elas, Web semântica, Protégé, E-cidade. Na seção 4 é apresentado o modelo RDF-Schema base para a ontologia para a integração de dados. Na seção 5 são apresentadas as conclusões obtidas com o desenvolvimento do trabalho, são apresentados também os trabalhos futuros para a continuação do desenvolvimento da ontologia. Por fim, na seção 6 são listadas as referências utilizadas para o desenvolvimento do presente trabalho.

\section{TRABALHOS RELACIONADOS}

Diversos trabalhos em Cidades Inteligentes vêm sendo desenvolvidos com o foco de construção de ontologias para construção de aplicações, integração de dados, dentre outras atividades. Segundo [3], a ontologia tem um papel importante no gerenciamento de Cidades Inteligentes para integração de dados, suporte a decisão, dentre outros. No caso deste trabalho, os autores consideram que esse conhecimento de domínio administrativo pode ser coletado de um grande número de especialistas. Assim sendo, no artigo é apresentado um modelo RDF-Schema proposto para construção de uma ontologia que poderá ser construída por pessoas que não tenham formação em computação.

Em [12] é apresentada uma metodologia para construção de ontologia no contexto de integração de dados. Tal trabalho nos auxiliou para guiar o processo metodológico deste trabalho. Ao final, os autores apresentam um estudo de caso realizado no domínio de consumo de energia e emissão de carbono em edifícios.

Em [8], os autores afirmam que há um baixo impacto nas aplicações de cidades inteligentes nas áreas de energia e transporte, e que há outras áreas nos quais o impacto de cidades inteligentes também é limitado. Ainda, argumentam que o impacto das aplicações dependem em primeiro lugar no conhecimento no qual se baseiam (ontologia) e, em segundo lugar nas tecnologias inteligentes e nas caracterís- ticas das aplicações. Assim sendo, os autores apresentam uma ontologia geral para cidades inteligentes, baseando-se em trabalhos com as mais citadas definições de cidades inteligentes. Daí, os autores avaliam como as ontologias de uma amostra de aplicações de cidades inteligentes se ajustam a essa ontologia geral.

Em [6] é definida uma ontologia genérica e reusável para representar definições de Indicadores Globais de Cidades e sua instanciação na Web Semântica pelas cidades. A ontologia combina, adapta e estende ontologias de fundamentação existentes. Tais ontologias existentes reusadas foram de localização de lugares ${ }^{1}$, de unidades de medidas $^{2}$, de estatísticas e indicadores de cidades ${ }^{3}$, de proveniência da informação $^{4}$ e de relações temporais ${ }^{5}$. Ainda, os autores combinam essas ontologias para construir uma ontologia de validação de informações ${ }^{6}$ e uma ontologia de confiança para avaliar a confiança da fonte da informação entre dois agentes ${ }^{7}$. Por fim, a ontologia de Indicadores Globais de Cidades (GCI Global City Indicator) também foi construída, que consolida toda a proposta dos autores ${ }^{8}$.

\section{TECNOLOGIAS E FERRAMENTAS UTI- LIZADAS}

\subsection{Web Semântica}

O principal acesso da população às informações governamentais abertas será via Web. Os primeiros sítios da Web disponibilizavam informações em HTML, ou seja, em documentos hipertexto. Tais sítios fizeram parte da primeira geração da Web, na qual o ser humano escrevia todas as páginas HTML. Já a segunda geração da Web se caracterizou pela geração automática de código HTML pelas máquinas, e o uso frequente de páginas HTML ativas. Ambas as gerações foram idealizadas para o processamento humano direto - leitura, busca e preenchimento de formulários. A terceira geração da Web, ainda em desenvolvimento, é chamada Web Semântica [2, 5], e visa dispor informações na Web de maneira processável pelos computadores. A Web Semântica possibilita serviços inteligentes, tais como agentes intermediadores de informações, agentes de busca, filtros de informações, etc. Tais serviços inteligentes na Web do Conhecimento podem melhorar as versões correntes dos serviços já oferecidos, o que atende os preceitos de Cidade Inteligente. Deve ser observado que a Web Semântica, e de maneira análoga alguns sistemas em uma Cidade Inteligente, somente se torna possível quando níveis mais profundos de interoperabilidade são estabelecidos. Para isso, padrões devem ser definidos não somente para a forma sintática dos documentos, mas também para seus conteúdos semânticos.

\footnotetext{
${ }^{1}$ Ontologia Geonames, disponível em http://www . geonames.org/ontology/

${ }^{2}$ Disponível em http://www.wurvoc.org/vocabularies/ om-1.8/

${ }^{3}$ Disponível em http://ontology.eil.utoronto.ca/

${ }^{4}$ Disponível em http://www.w3.org/ns/prov

${ }^{5}$ Disponível em http://www.w3.org/2006/time

${ }^{6}$ Disponível em http://ontology.eil.utoronto.ca/kp. owl

${ }^{7}$ disponível emhttp://ontology.eil.utoronto.ca/trust. owl

${ }^{8} \mathrm{~A}$ ontologia GCI está disponível em http://ontology. eil.utoronto.ca/GCI-v1.owl
} 
Tal interoperabilidade semântica é facilitada pelos esforços de padronização da W3C, que inclui o RDF (Resource Description Framework), o Esquema RDF, e a linguagem de ontologia para Web OWL (Ontology Web Language) [16]. A intenção do Esquema RDF é prover um modelo de dados básico do tipo OAV (Objeto-Atributo-Valor) para meta-dados. Assim, o Esquema RDF permite a definição de um vocabulário particular que pode ser usado pelos atributos RDF. A OWL é uma linguagem da Web Semântica projetada para representar conhecimento rico e complexo sobre coisas, grupos de coisas, e relações entre as coisas. Ainda, OWL/RDF é uma linguagem computacional baseada em lógica tal que o conhecimento expressado em OWL pode ser explorado por programas de computadores, p. ex., para verificar a consistência do conhecimento ou tornar explícito um conhecimento implicito. Documentos OWL/RDF, conhecidos como ontologias, podem ser publicados na Web e pode referenciar ou ser referenciado por outras ontologias OWL. Neste trabalho, utilizamos a ferramenta Enterprise Architect [14] para criar o modelo RDF-Schema que será utilizado na ferramenta Protégé [15] para a construção de uma ontologia para integração de dados de cidadãos.

\subsection{O E-Cidade}

O objetivo do software público E-Cidade é informatizar a gestão dos Municípios Brasileiros de forma integrada. Essa informatização contempla a integração entre diversos entes municipais, dos quais podem ser citados a Prefeitura Municipal e suas secretarias, a Câmara Municipal, Autarquias, Fundações e outros [13]. O software possui nove módulos: Módulo Educação, Módulo Saúde, Módulo Financeiro, Módulo Patrimonial, Módulo Cidadão, Módulo Gestor, Módulo Recursos Humanos, Módulo BI e Módulo Geoprocessamento. Como o software foi concebido para uma integração entre os módulos, foi utilizado o conhecimento implícito no software, presente no Banco de Dados da aplicação, para verificar quais informações do cidadão são armazenadas. A partir daí, um modelo RDF-Schema foi criado para auxiliar a construção de uma ontologia que apoiará processos de integração de dados em diversas prefeituras. Isso é importante pois muitas prefeituras possuem sistemas legados, que não foram concebidos com o princípio de integração do E-Cidade.

\section{UM MODELO RDF-SCHEMA BASE DE UMA ONTOLOGIA PARA INTEGRAÇÃO DE DADOS DE MUNICÍPIOS}

Em [1] é ilustrado o modelo RDF-Schema construído na ferramenta Enterprise Architect [14] e validado na ferramenta Protégé [15]. Este modelo foi gerado a partir da criação de um modelo de classe de domínio feito com referência às informações contidas no módulo saúde do software E-cidade [13].

O modelo construído neste trabalho, por ser em formato OWL/RDF, segue as definições da linguagem em questão, no qual temos três recursos essenciais em sua estrutura: os rdfs:Resource, que representam o conjunto de recursos do modelo básico da OWL/RDF; as rdfs:Property, que representam as propriedades da OWL/RDF, ou seja, subconjuntos de recursos; e as rdfs:Class, que são classes que podem representar diversos tipos de recursos, o que diferencia o RDF das definições de classes utilizadas em outros paradig- mas de programação. O modelo RDF-Schema é um modelo para OWL/RDF, que utiliza propriedades específicas RDF para representar os relacionamentos entre seus recursos. Outra característica existente no modelo RDF-Schema deste trabalho é a utilização da propriedade de restrição rdfs:SubPropertyOf, a qual impede a criação de laços entre as classes, gerando assim um modelo OWL/RDF consistente. O domínio em questão abrange um extenso número de informações para o sistema, o que torna o nosso modelo RDFSchema complexo, exigindo que seja feita uma simplificação para a construção da ontologia.

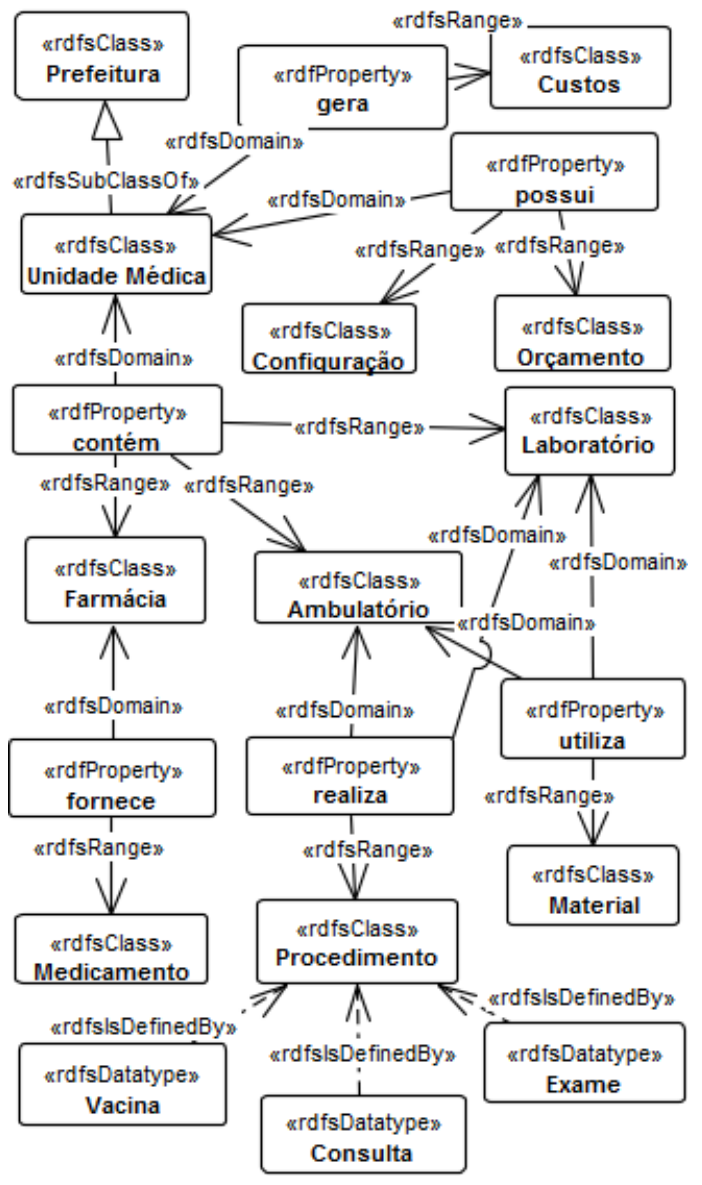

Figura 1: Modelo RDF-Schema reduzido.

Na Figura 1 é apresentado um modelo reduzido do modelo RDF-Schema [1] desenvolvido para o módulo saúde do software e-cidade, como o modelo RDF-schema desenvolvido no presente trabalho possui um número muito grande de classes e tipos de dados a Figura 1 é utilizada para demonstrar a estrutura base do mesmo. No modelo [1], assim como na Figura 1, está representada a estrutura de uma unidade médica relacionada a uma prefeitura. Foram definidas classes, subclasses, propriedades e tipos de dados que estão diretamente ligados a classes. Seguindo a convenção da linguagem OWL/RDF, as classes e os tipos de dados são descritos com a primeira letra maiúscula, as propriedades descritas com letra minúscula. As ligações entre classes e subclasses são demonstradas por linhas contínuas e uma seta preenchida, 
as ligações entre classes e propriedades utilizam linhas contínuas e setas marcadas e por fim, as ligações entre as classes e os tipos de dados são representadas por setas tracejadas e setas marcadas. Todos os dados relacionados a uma subclasse são automaticamente agregados a sua classe superior, o que possibilita que cada subclasse tenha suas atribuições específicas sem alterar a validação da estrutura do modelo como um todo.

Um modelo RDF-Schema como o exibido na Figura 1 permite que, ao construir a ontologia a partir dele, os dados cadastrados e utilizados dentro da estrutura de uma cidade possam ser unificados e tratados de uma maneira mais simples, por exemplo a classe procedimento - uma secretaria de saúde pode cadastrar um procedimento de uma maneira diferente da utilizada em um hospital desta mesma cidade. A classe procedimento armazena e padroniza a maneira como eles serão identificados, essa padronização está representada nos tipos de dados definidos pela classe, sendo eles Vacina, Consulta e Exame. Todas as classes podem ter seus tipos de dados estendidos e definidos. O modelo apresentado neste trabalho é uma versão reduzida do modelo RDF-Schema em [1], que abrange inteiramente o módulo de saúde do software E-cidade, apresentando inclusive as informações que não são utilizadas na linguagem OWL/RDF, estas informações estão representadas em classes sem relacionamentos.Este tipo de modelo, assim como a linguagem OWL/RDF, é baseado em lógica o que torna simples o entendimento e a obtenção das informações exatas para a criação da ontologia e outras análises feitas sobre o domínio especificado.

\section{CONCLUSÕES}

O objetivo deste trabalho foi construir um modelo de dados em RDF, um modelo RDF-schema, para (i) facilitar a interoperabiidade de informações entre sistemas em Cidades Inteligentes na área de saúde e (ii) facilitar a construção futura de uma ontologia para cidades inteligentes brasileiras. Deve-se considerar ainda que foram tratados aqui somente os níveis de dados referentes ao módulo saúde do software ecidade. Como trabalhos futuros pretendemos criar uma ontologia baseada no modelo RDF-Schema apresentado em [1] e a validação da mesma por especialistas, juntamente com a extensão da ontologia para todos os módulos do software E-cidade.

\section{AGRADECIMENTOS}

Os autores gostariam de agradecer ao suporte financeiro do CNPq através do edital Universal - MCTI/CNPq Número $14 / 2013$.

\section{REFERENCIAS}

[1] Bernardini, F., Ferronato, A., Pires, P. Modelo rdf-schema para a área de saúde governamental, 2016. Disponível em http://www.pxsti.com.br/artigo/ Modelo_FinalSaude.pdf. Acessado em 01 de fevereiro de 2016.

[2] BERNERS-LEE, T., HENDLER, J., AND LASSILA, O. The semantic web. Scientific American (2001).

[3] CHUNG, T., XU, B., ZHANG, P., TAN, Y., ZHU, P., ANd WUBUlihasimu, A. Semantic Technology, vol. 8388 - LNCS. Springer, 2014, ch. Constructing City Ontology from Expert for Smart City Management, pp. 187-194.

[4] DAMERI, R. Defining an evaluation framework for digital cities implementation. In IEEE Internation Conference on Information Society (i-Society) (2012).

[5] DECKER, S., HARMELEN, F., BROEKSTRA, J., ERDMANN, M., FENSEL, D., HORROCKS, I., KLEIN, M., AND MELNIK., S. The semantic web - on the respective roles of $\mathrm{xml}$ and rdf. IEEE Internet Computing 4 (2000).

[6] FOX, M. A foundation ontology for global city indicators. Working Paper N. 3, 2013. Disponível em http://eil.utoronto.ca/wp-content/uploads/ smartcities/papers/GCI-Foundation-Ontology.pdf. Acessado em 01 de fevereiro de 2016.

[7] GIFFINGER, R., AND GUDRUN, H. Smart cities ranking: an effective instrument for the positioning of cities? ACE: Architecture, City \& Environ. 4, 12 (2010), 7-25.

[8] Komninos, N., Bratsas, C., Kakderi, C., And TsARChOpOulos, P. Smart city ontologies: Improving the effectiveness of smart city applications. Journal of Smart Cities 1, 1 (2015). Disponível em http://jsc. whioce.com/index.php/JSC/article/view/01001. Acessado em 01 de fevereiro de 2016.

[9] LEE, J. H., PHAALB, R., AND LEE, S. An integrated service-device-technology roadmap for smart city development. Technological Forecasting and Social Change 80, 2 (2013), 286-306.

[10] LEE, S., HAN, J., LEEM, Y., AND YIGITCANLAR, T. Towards ubiquitous city: concept, planning, and experiences in the Republic of Korea. IGI Global, 2008, ch. Knowledge-based Urban Development: Planning and Applications in the Information Era.

[11] Marini, V. Comunicação pessoal, 2013.

[12] Nemirovski, G., Nolle, A., Álvaro Sicilia, Ballarini, I., ANd Corado, V. Data integration driven ontology design, case study smart city. In Proc. 3rd International Conf. on Web Intelligence, Mining and Semantics (2013). Disponível em http://www.semanco-project.eu/index_htm_files/ WIMS2013.pdf. Acessado em 01 de fevereiro de 2016.

[13] Portal do Software Público Brasileiro Ministério do Planejamento, OrÇamento e Gestão. e-Cidade, 2015. Disponível em https: //softwarepublico.gov.br/social/e-cidade/. Acessado em 01 de fevereiro de 2016.

[14] Sparx Systems. Enterprise architect: A plataform that accelerates and integrates software - trial version, 2015. Disponível em

http://www.sparxsystems.com.au/. Acessado em 01 de fevereiro de 2016

[15] Stanford Center for Biomedical Informatics Research. Protégé: A free, open-source ontology editor and framework for building intelligent systems, 2015. Disponível em http://protege.stanford.edu/. Acessado em 01 de fevereiro de 2016.

[16] W3C Semantic Web - OWL Working Group. Web ontology language (owl), 2012. Disponível em https://www.w3.org/2001/sw/wiki/OWL. Acessado em 01 de fevereiro de 2016 